\title{
Erratum: Onset of low Prandtl number thermal convection in thin spherical shells [Phys. Rev. Fluids 3, 024801 (2018)]
}

\author{
F. Garcia, F. R. N. Chambers, and A. L. Watts \\ (Received 9 March 2018; published 18 May 2018)
}

DOI: 10.1103/PhysRevFluids.3.059901

We have performed the linear stability analysis in thin rotating spherical shell thermal convection models at a low Prandtl number. The results have been used to extrapolate to several astrophysical scenarios, namely, the sun, cooling white dwarfs, and accreting neutron stars. Unfortunately there was an error in the estimation of the kinematic viscosity for the case of accreting neutron star oceans, which we correct here. This erratum concerns only a small part of the paper in Sec. VI involving one column in Tables II and III and Fig. 8. The changes are discussed in the following.

Tables I and II (corresponding to Tables II and III of the original paper) provide the correct values for the accreting neutron star ocean scenario. A valid estimation for the kinematic viscosity $v$ is $v \sim$ $1 \mathrm{~cm}^{2} \mathrm{~s}^{-1}$ rather than $v \sim 10^{6} \mathrm{~cm}^{2} \mathrm{~s}^{-1}$ stated in the original paper. The new value of $v$ and slightly amended values for the thermal conductivity $\kappa=10^{3}-10^{5} \mathrm{~cm}^{2} \mathrm{~s}^{-1}$ and gap width $d=10^{2}-10^{4} \mathrm{~cm}$ ( $\kappa=10^{6} \mathrm{~cm}^{2} \mathrm{~s}^{-1}$ and $d=10^{3}-10^{4} \mathrm{~cm}$ were supposed in our paper) give rise to new estimations of the parameters [Prandtl number (Pr) and Taylor numbers (Ta)], critical parameters [critical Rayleigh number $\left(\operatorname{Ra}_{c}\right)$, wave-number $m_{c}$, and frequency $\left.\omega_{c}\right]$ and typical time-scale $P=\left(2 \pi m_{c} /\left|\omega_{c}\right|\right) d^{2} / \nu$. They are shown in Table II.

As a result of the new viscosity the Prandtl number of an accreting neutron star ocean becomes very low $\operatorname{Pr} \in\left[10^{-5}, 10^{-3}\right]$ and the Taylor number moderately large $\mathrm{Ta} \in\left[10^{12}, 10^{20}\right]$, and thus our linear stability analysis falls quite close to this regime. This is shown in Fig. 1 (corresponding to Fig. 8 of our paper). At this regime, according to our extrapolation in the original paper for the critical Pr marking the transition among modes the most feasible mode is of equatorial (EA) type instead of spiralling (the result reported in the original paper). However it may also be of polar (P) type due to the new estimation of very low Pr and moderately large Ta.

Comparing the new Table II (EA and P modes) with Table III of our paper, the new critical parameters and time scales have not increased substantially. For instance, we have obtained time scales on the order of 10-100 s for EA modes and on the order of 1-10 s for P modes. These are similar to those obtained in the original paper for spiralling modes at $\operatorname{Pr}=10^{2}$ using the estimations

TABLE I. Physical parameters corresponding to an accreting neutron star ocean. Thermal diffusivity $\kappa$, kinematic viscosity $\nu$, layer width $d$, and rotation rate $\Omega$. Values are taken from Refs. [2-4].

\begin{tabular}{lc}
\hline \hline Property & $\begin{array}{c}\text { Accreting neutron } \\
\text { star ocean }\end{array}$ \\
\hline$\kappa\left(\mathrm{cm}^{2} \mathrm{~s}^{-1}\right)$ & $10^{3}-10^{5}$ \\
$\nu\left(\mathrm{cm}^{2} \mathrm{~s}^{-1}\right)$ & $10^{0}$ \\
$d(\mathrm{~cm})$ & $10^{2}-10^{4}$ \\
$\Omega\left(\mathrm{s}^{-1}\right)$ & $10^{2}$ \\
\hline \hline
\end{tabular}


TABLE II. Estimation of the critical parameters and a typical time-scale $P=$ $\left(2 \pi m_{c} /\left|\omega_{c}\right|\right) d^{2} / v$ in seconds (this is the time scale associated with the drifting phase velocity as computed in the rotating frame) for the Sun, a white dwarf, and a neutron star depending on the type of mode, spiralling (SC), EA, P, or symmetric polar (SP). All of them could be preferred depending on appropriate particular choice of Pr. This is indicated by the critical Pr at the P/EA and EA/SC transitions, which are also shown.

\begin{tabular}{|c|c|c|}
\hline Type of mode & Property & $\begin{array}{l}\text { Accreting neutron } \\
\text { star ocean }\end{array}$ \\
\hline & $\mathrm{Ta}$ & $10^{12}-10^{20}$ \\
\hline & $\operatorname{Pr}$ & $10^{-5}-10^{-3}$ \\
\hline & $\operatorname{Pr}_{\mathrm{P} / \mathrm{EA}}$ & $10^{-4}-10^{-6}$ \\
\hline & $\operatorname{Pr}_{\mathrm{EA} / \mathrm{SC}}$ & $10^{-2}-10^{-4}$ \\
\hline & $\mathrm{Ra}_{\mathrm{c}}$ & $10^{6}-10^{10}$ \\
\hline & $\left|\omega_{c}\right|$ & $10^{4}-10^{7}$ \\
\hline \multirow[t]{4}{*}{ SP } & $m_{c}$ & $10^{2}-10^{4}$ \\
\hline & $P$ (years) & $10^{-5}-10^{-2}$ \\
\hline & $\mathrm{Ra}_{\mathrm{c}}$ & $10^{5}-10^{10}$ \\
\hline & $\left|\omega_{c}\right|$ & $10^{5}-10^{9}$ \\
\hline \multirow[t]{4}{*}{ EA } & $m_{c}$ & $10^{2}-10^{3}$ \\
\hline & $P$ (years) & $10^{-6}-10^{-5}$ \\
\hline & $\mathrm{Ra}_{\mathrm{c}}$ & \\
\hline & $\left|\omega_{c}\right|$ & $10^{6}-10^{9}$ \\
\hline \multirow[t]{2}{*}{$\mathrm{P}$} & $m_{c}$ & $10^{1}-10^{3}$ \\
\hline & $P$ (years) & $10^{-7}-10^{-6}$ \\
\hline
\end{tabular}

provided in Ref. [1], but this should not be surprising because two parameters ( $\mathrm{Pr}$ and $\mathrm{Ta}$ ) have changed.

The main conclusion of this erratum is that accreting neutron star oceans are characterized by low Prandtl numbers rather than larger ones as for the cooling white dwarfs and the sun scenarios. Thus low Prandtl numbers in thin shells (the regime in our paper addresses) should be considered for their modeling. Then, because the main difference between neutron star oceans and white dwarfs or solar convective zones is now their width, and different Ta regimes, giving rise to very different time scales, should be expected. As concluded in our original paper these time scales could be linked with some observational properties in the three astrophysical scenarios considered.

The authors acknowledge support from ERC Starting Grant No. 639217 CSINEUTRONSTAR (PI Watts). They wish to thank A. Cumming for identifying the error in the determination of the kinematic viscosity of an accreting neutron star ocean. 

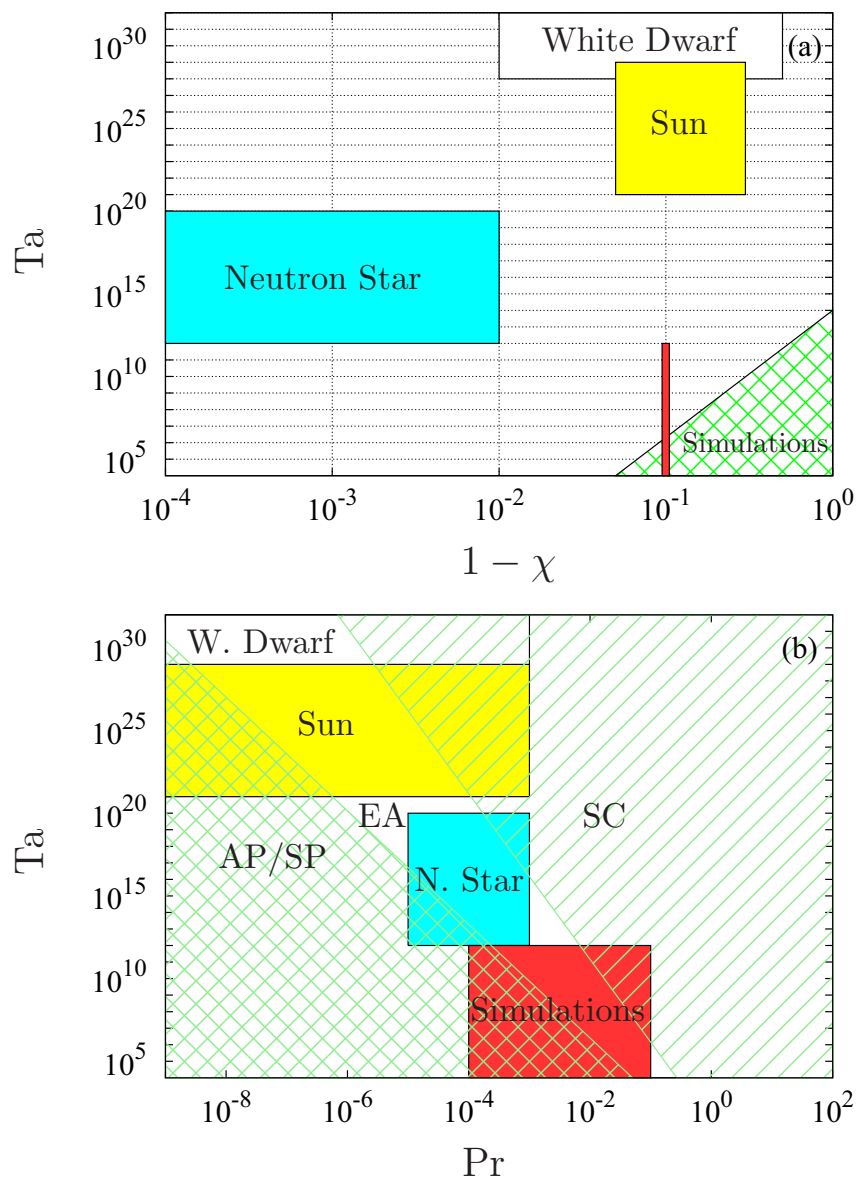

FIG. 1. (a) Estimates of Ta and $1-\chi$ for stellar convective oceans and parameter values of the current (red) and previous (green) numerical studies of thermal convection, which are clearly still far from stellar conditions. (b) The same as (a) but for Ta and Pr. The estimated region of stability of the different convective instabilities SC (with stripes), EA (plain), and antisymmetric polar (AP)/SP (cross hatched) is also shown.

[1] K. Zhang, Spiralling columnar convection in rapidly rotating spherical fluid shells, J. Fluid Mech. 236, 535 (1992).

[2] R. Nandkumar and C. J. Pethick, Transport coefficients of dense matter in the liquid metal regime, Mon. Not. R. Astron. Soc. 209, 511 (1984).

[3] D. G. Yakovlev and V. A. Urpin, Thermal and electrical conductivity in white dwarfs and neutron stars, Soviet Astron. 24, 303 (1980).

[4] A. L. Watts, Thermonuclear burst oscillations, Annu. Rev. Astron. Astrophys. 50, 609 (2012). 